Relations industrielles

Industrial Relations

\title{
Les relations industrielles : un point de vue international Industrial Relations : An International Viewpoint
}

\section{J. De Givry}

Volume 29, numéro 2, 1974

URI : https://id.erudit.org/iderudit/028502ar

DOI : https://doi.org/10.7202/028502ar

Aller au sommaire du numéro

Éditeur(s)

Département des relations industrielles de l'Université Laval

ISSN

0034-379X (imprimé)

1703-8138 (numérique)

Découvrir la revue

Citer cet article

De Givry, J. (1974). Les relations industrielles : un point de vue international. Relations industrielles / Industrial Relations, 29(2), 255-271.

https://doi.org/10.7202/028502ar
Résumé de l'article

En abordant les relations industrielles d'un point de vue international, l'auteur traite d'abord de problèmes ayant trait aux relations professionnelles dans le cadre de l'entreprise et s'appuie ensuite sur l'expérience des pays industrialisés et plus spécifiquement des pays européens telle qu'elle ressort notamment de la deuxième session de la Conférence régionale européenne de VOIT.
Tous droits réservés @ C Département des relations industrielles de l'Université Laval, 1974
Ce document est protégé par la loi sur le droit d'auteur. L'utilisation des services d'Érudit (y compris la reproduction) est assujettie à sa politique d'utilisation que vous pouvez consulter en ligne.

https://apropos.erudit.org/fr/usagers/politique-dutilisation/ 


\title{
Les relations industrielles: un point de vue international
}

\author{
J. de Givry
}

En abordant les relations industrielles d'un point de vue international, l'auteur traite d'abord de problèmes ayant trait aux relations professionnelles dans le cadre de l'entreprise et s'appuie ensuite sur l'expérience des pays industrialisés et plus spécifiquement des pays européens telle qu'elle ressort notamment de la deuxième session de la Conférence régionale européenne de l'OIT.

Les situations sont si diverses d'un pays à un autre, d'une branche d'activité économique à une autre, d'une entreprise à une autre, que toute généralisation est immédiatement sujette à caution. De prime abord, on serait même tenté de penser qu'étant donné les énormes différences que l'on peut constater entre les systèmes de relations industrielles des divers pays du monde et la grande variété des facteurs - politiques, économiques et culturels - qui influent sur ces systèmes, il n'est pas possible de déceler des tendances générales.

Et pourtant, cette réaction négative ne me paraît pas justifiée. De nos jours, infiniment rares sont les pays qui vivent encore isolés. Partout où règne la liberté d'expression, les idées franchissent les frontières et les réunions convoquées par l'Organisation internationale du travail où des représentants des gouvernements, des employeurs et des travailleurs venus des quatre coins du monde se rencontrent régulièrement, offrent en particulier une occasion unique de découvrir les problèmes

DE GIVRY, J. Chef du département du développement des institutions sociales, Bureau international du travail, Genève, Suisse.

* Exposé prononcé à la Journée d'études organisée à l'occasion du dixième anniversaire de la Corporation des conseillers en relations industrielles du Québec, Montréal, 25 janvier 1974. 
et les courants communs. De plus, lorsque ces assemblées forgent des instruments de portée internationale, comme c'est le cas pour les conventions et les recommandations internationales adoptées par la Conférence internationale du travail, il n'est pas vain d'affirmer que ces décisions, qui sont acquises à la majorité des deux tiers, représentent un consensus de l'opinion internationale sur certains problèmes essentiels de politique sociale, obtenu après une série de discussions des plus approfondies.

Voilà pourquoi, en dépit des écarts considérables que l'on peut noter entre les systèmes de relations professionnelles des différents pays du monde et malgré la diversité des facteurs qui sont en jeu, ceux qui observent la scène internationale ont le devoir non seulement de procéder à une analyse comparative des différents systèmes de relations professionnelles, mais aussi de retenir ou de souligner les tendances générales et les problèmes qu'ils estiment particulièrement significatifs pour l'évolution ultérieure de ces systèmes. Il est vrai que les tendances ainsi définies ressemblent souvent à ces vues aériennes où la forêt cache les arbres. Cependant, si l'on consent à considérer ces tendances plutôt comme des bases de discussion que comme des déductions rigides et définitives, elles peuvent peut-être éclairer la réflexion de ceux qui sont, comme vous, engagés dans l'action.

Cela dit, étant donné l'ampleur du sujet, il m'est apparu nécessaire de faire des choix. D'une part, je me limiterai à traiter de problèmes ayant trait aux relations industrielles dans le cadre de l'entreprise. D'autre part, je m'appuierai surtout sur l'expérience des pays industrialisés et plus spécialement des pays européens, telle qu'elle ressort notamment de la deuxième session de la Conférence régionale européenne de l'OIT qui vient de terminer ses travaux à Genève.

\section{L'ÉVOLUTION DU CADRE INSTITUTIONNEL DES RELATIONS ENTRE EMPLOYEURS ET TRAVAILLEURS AU NIVEAU DE L'ENTREPRISE}

Avant de aégager certaines tendances d'actualité en matière de relations entre employeurs et travailleurs au niveau de l'entreprise, je voudrais rappeler brièvement les trois axes principaux autour desquels l'évolution du cadre institutionnel de ces relations me paraît s'être déroulée depuis la deuxième Guerre mondiale.

Cette évolution s'est effectuée dans le cadre de trois courants principaux qui, loin d'être indépendants les uns des autres ou imperméables les uns aux autres, s'entrecroisent en fait constamment. 
Un premier courant, d'origine essentiellement patronale, tend à améliorer les relations dans l'entreprise en mettant l'accent sur la communauté d'objectifs qui doit unir tous les membres d'une même entreprise et sur les moyens destinés à susciter le plus haut degré de collaboration de chacun à l'œuvre commune. La recherche de ces moyens a été facilitée par le progrès des connaissances en ce qui concerne, d'une part, les besoins et les aspirations de l'homme au travail - et l'apport des sciences sociales du travail a été ici essentiel - et, d'autre part, les techniques de direction des entreprises. Il est incontestable qu'une politique et du personnel moderne, avec tout ce qu'elle comporte sur le plan de la formation, des méthodes de commandement ou de la communication entre les hommes, et que des méthodes d'organisation des entreprises faisant une large place à la décentralisation des responsablités, notamment dans le cadre de ce qu'on appelle la direction par objectifs, peuvent grandement contribuer à créer dans l'entreprise un climat qui soit à la mesure de l'homme.

Dans un second courant - d'origine essentiellement politique s'inscrivent les mesures qui ont remis en cause les structures de l'entreprise privée, soit en abolissant purement et simplement la propriété privée des moyens de production et en mettant en place de nouvelles structures économiques dans le cadre de régimes socialistes à économie planifiée, soit en transformant plus ou moins profondément ces structures de manière à faire participer les travailleurs à la direction des entreprises. L'introduction de systèmes de cogestion, voire d'autogestion des entreprises, les débats autour de la réforme de l'entreprise et des diverses formules de représentations des travailleurs au conseil d'administration des entreprises sont autant de manifestations de l'intérêt porté à des réformes qui tendent à modifier la structure de l'entreprise privée à la source même où s'exerce son autorité.

Enfin, dans un troisième courant, d'inspiration principalement syndicale, on peut regrouper toutes les mesures qui, soit par la voie législative, soit par la voie contractuelle, et l'action syndicale quotidienne visent moins à modifier l'entreprise dans ses structures de base qu'à réglementer le comportement de ses dirigeants de manière à limiter leur liberté d'action par toute une série de procédures et d'institutions faisant contrepoids à leur autorité et empêchant l'arbitraire. C'est l'application au plan professionnel du fameux principe posé par Montesquieu dans l'Esprit des Lois, selon lequel «Quiconque a du pouvoir est porté à en abuser. Il faut donc que par une juste disposition des choses le pouvoir arrête le pouvoir ». Le droit d'organisation et de négociation 
collective pour les travailleurs, avec son corollaire naturel qui est le droit de grève, le droit pour leurs représentants de bénéficier d'une protection spéciale dans l'exercice de leurs fonctions, ainsi que des facilités nécessaires pour remplir leur mandat, le droit des travailleurs de soumettre des réclamations sans qu'il en résulte pour les intéressés un préjudice quelconque, leur droit à ne pas être licenciés sans un motif valable sont autant d'éléments d'un véritable statut des travailleurs dans l'entreprise dont on a dégagé peu à peu les contours et qui accorde aux travailleurs à l'égard de l'employeur des garanties pouvant être mises en parallèle avec celles que contiennent les chartes et les constitutions politiques qui définissent les droits fondamentaux des citoyens en face de la puissance publique. L'OIT s'est pour sa part attelée, au cours de ces dernières années, à définir au plan international certains éléments de base d'un tel statut du travailleur dans l'entreprise.

\section{LES TENDANCES D'ACTUALITÉ}

En étudiant la pratique des relations professionnelles et les institutions qui s'y rattachent, je ne peux m'empêcher d'être frappé par le fait que certaines aspirations ou exigences essentielles semblent se faire jour dans le cadre de systèmes très différents. Je les grouperai autour de quatre mots clefs : sécurité, participation, justification et formation.

\section{Pour une plus grande sécurité}

Dans le monde en voie de profonde transformation qui est aujourd'hui le nôtre, il n'est guère surprenant que le souci de sécurité ait pris une place prédominante dans le domaine des relations industrielles.

Aussi, une tendance accrue à la sécurité de l'emploi et du revenu se manifeste-t-elle dans la plupart des pays. Le principe selon lequel un travailleur ne devrait pas être arbitrairement privé de son emploi est aujourd'hui définitivement consacré sur le plan international. En 1963, la Conférence internationale du travail a adopté la Recommandation No. 119 sur la cessation de la relation de travail, laquelle stipule notamment qu' «aucun licenciement ne devrait intervenir saris qu'il existe un motif valable de licenciement...». Du point de vue de la philosophie des relations professionnelles, une étape considérable a été franchie par rapport au vieux principe selon lequel tout travailleur recruté par un employeur sous le régime d'un contrat de durée indéterminée pouvait voir ce contrat résilié par la volonté de l'une ou l'autre 
des parties moyennant un préavis d'une certaine durée. Il est vrai que la Recommandation No. 119 prévoit également que, dans les cas de cessation de l'emploi - à l'exception des cas de faute grave - il y aurait lieu de donner un "préavis d'une durée raisonnable ». Il convient cependant de souligner que cette Recommandation va beaucoup plus loin puisqu'elle implique que la simple notification du préavis ne suffit pas et que le préavis ne peut être signifié que pour une "raison valable ». Cet instrument stipule en outre que, par voie de conséquence, si le travailleur «estime avoir fait l'objet d'une mesure de licenciement injustifiée », il devrait avoir le droit de recourir contre cette mesure dans un délai raisonnable «devant un organisme institué en vertu d'une convention collective ou devant un organisme impartial tel qu'un tribunal, un arbitre, une commission d'arbitrage ou un organisme similaire , et que cet organisme "devrait être habilité à examiner les motifs invoqués pour justifier le licenciement, ainsi que les autres circonstances pertinentes et à se prononcer sur la justification du licenciement ».

La reconnaissance du principe du «motif valable * me semble extrêmement importante pour assurer que le travailleur jouisse d'un réel statut dans l'entreprise où il est occupé. Toutefois, on ne saurait relever là aucune trace de démagogie. Aux termes de la Recommandation No. 119 , ce motif valable peut «être lié à l'aptitude ou à la conduite du travailleur ou fondé sur les nécessités du fonctionnement de l'entreprise, de l'établissement ou du service ».

Si la reconnaissance du principe de la justification du licenciement est essentiel pour garantir la dignité du salarié partie à la relation de travail, il n'en reste pas moins que l'accroissement de la mobilité de la main-d'œuvre tend à devenir l'un des traits caractéristiques de notre société industrielle parce que l'on admet de plus en plus que le progrès technique et la croissance économique exigent une mobilité de la maind'œuvre plus grande que par le passé. Sécurité n'est donc pas synonyme de stabilité ! Au fur et à mesure que certains emplois deviennent superflus ou périmés, il n'est plus possible de protéger à tout prix un emploi particulier. Ce qu'il faut, c'est rechercher la sécurité à travers les changements que nécessite la croissance plutôt que de maintenir à tout prix le statut quo s'il doit être source de stagnation et entraver une politique de progrès pour le plus grand nombre. Dans ces conditions, les efforts déployés pour assurer une sécurité accrue vont surtout se concentrer sur la protection du revenu des travailleurs et sur les mesures à prendre pour favoriser leur réemploi, plutôt que sur les moyens de les retenir dans l'emploi qu'ils exercent. Un principe essentiel du point de vue de 
la justice sociale se fait maintenant jour à ce propos, à savoir que si des réductions de personnel peuvent être justifiées en tant que facteur de progrès économique, les travailleurs affectés par ces réductions devraient bénéficier d'une attention particulière. La Recommandation de l'OIT déclare que les salariés ont besoin d'une certaine forme de " protection du revenu », ainsi que d'une aide pour trouver un nouvel emploi et, le cas échéant, pour acquérir les qualifications nouvelles propres à faciliter ce réemploi. Dans le véritable combat industriel qui est celui des sociétés modernes, dont des groupes de travailleurs se trouvent soudain être les victimes, pour la seule raison qu'ils sont «de trop > ou que leurs qualifications se trouvent dépassées, c'est un devoir pour toute société qui se veut humaine de faire jouer à plein la solidarité professionnelle et nationale, d'une manière analogue à celle par laquelle, au sortir d'une guerre, elle récompensait ses combattants en déclarant qu' «ils ont des droits sur nous». La société tout entière devant bénéficier du progrès économique, il n'est que juste qu'elle assume également les coûts sociaux de ce progrès; tel est le nouveau principe qui, sur le plan de la justice sociale, paraît désormais s'affirmer. Il est significatif de constater que les deux questions techniques qui avaient été retenues par le Conseil d'administration du BIT pour figurer à l'ordre du jour de la deuxième Conférence régionale européenne de l'OIT (Genève, janvier 1974) aient précisément eu trait l'une à la "sécurité des revenus en Europe face aux changements structuraux " et l'autre à «L'emploi en Europe - Quelques problèmes d'importance croissante » et que l'on ait notamment souligné à cet égard "la multiplication et la diversification des aides individuelles destinées à la conversion, à la réadaptation et aux transferts professionnels, au moyen desquelles les garanties de revenus concourent plus directement au succès de la politique de la main-d'œuvre $>1$.

\section{Vers une participation accrue}

En 1966, la Conférence internationale du travail a adopté à l'unanimité une Résolution précisant que «l'évolution des techniques et l'expansion de l'économie influent d'une manière toujours plus importante sur les conditions de vie et de travail > et que «dans divers pays ayant des structures économiques et sociales différentes, des efforts et des expériences ont été et sont faits pour permettre aux travailleurs

1 Deuxième Conférence régionale européenne, Genève, janvier 1974. Rapport III: La sécurité des revenus en Europe face aux changements structuraux. BIT Genève, 1974, p. 115. 
de participer aux décisions prises dans leur entreprise, surtout lorsque celles-ci affectent leur emploi et leurs conditions de travail et de vie \$. Dans ces conditions, la Conférence a demandé que soit entreprise une étude «des diverses manières en cours dans le monde pour faire participer les travailleurs aux décisions dans les entreprises».

A la suite de quoi, tant les services du BIT que l'Institut international d'études sociales, créé sous les auspices du BIT, ont entrepris des études approfondies dans un certain nombre de pays et une Réunion technique d'experts fut convoquée par le BIT en 1967 pour tirer quelques conclusions. Par la suite un Séminaire international fut organisé à Belgrade en 1969 et le Programme de I'OIT pour cette année prévoit la convocation d'un nouveau symposium international sur ce sujet qui se tiendra à Oslo en août 1974. De son côté la Conférence régionale européenne a souligné l'importance de la question en recommandant au Conseil d'administration de continuer à convoquer des réunions internationales permettant de faire le point de l'évolution de la situation dans ce domaine.

Le terme de «participation > est, certes, très ambigu et chargé d'émotivité. Il a acquis dans certains pays une nette coloration politique. Son seul emploi déclenche parfois des oppositions farouches. Je me souviens qu'à la session de la Conférence internationale du travail tenue en 1967, une des difficultés de dernière heure qui ont failli mettre en cause l'adoption à la quasi unanimité de la Recommandation No. 130 de l'OIT concernant l'examen des réclamations a tenu au fait qu'une disposition du projet soumis à la Conférence par la Commission chargée de l'examen de cette question prévoyait que les représentants des travailleurs devraient «participer » à l'élaboration de la politique du personnel ainsi qu'à la solution des questions d'ordre social se posant dans le cadre de l'entreprise. Les objections soulevées s'expliquent par le fait que, dans le langage des relations professionnelles, les termes «participer 》 et «participation» évoquent immédiatement dans de nombreux esprits le système de participation des travailleurs aux organismes de direction des entreprises. Et pourtant, ce n'est pas, loin de là, le seul moyen dont disposent les travailleurs pour infléchir les décisions adoptées dans l'entreprise, leur participation à ces décisions pouvant s'exercer de bien d'autres façons.

Tout en admettant qu'il n'est pas possible de parvenir à une définition du terme "participation 》 qui soit internationalement acceptable, étant donné que ce terme est interprété de façon différente selon 
les personnes, les pays et les époques, la Réunion Technique convoquée par l'OIT a néanmoins estimé que la notion de «participation des travailleurs aux décisions dans les entreprises » offre «un cadre général de référence qui, du point de vue d'une comparaison internationale, a l'avantage de mettre l'accent sur les différents types de décisions qui doivent être prises au sein de l'entreprise quel que soit le système économique en vigueur, ainsi que sur le degré de l'influence reconnue aux travailleurs à l'égard de telles décisions, compte tenu de la nature des problèmes à résoudre plutôt que sur les mécanismes très différents à travers lesquels ils peuvent influer sur lesdites décisions ». Envisagée dans cette perspective, l'expression \& participation des travailleurs aux décisions dans les entreprises permet de comparer « l'influence reconnue aux travailleurs au niveau de l'entreprise en ce qui concerne la préparation et l'adoption des décisions - ainsi que le contrôle de leur exécution - relevant de domaines très différents (tels que la fixation des salaires et des conditions de travail, la sécurité, la discipline et l'emploi, la main-d'œuvre et la formation professionnelle, les changements technologiques et l'organisation de la production ainsi que leurs conséquences sociales, les investissements, la programmation, etc.) et qui s'exercera à travers des mécanismes aussi différents que la consultation paritaire et les moyens de communication, la négociation collective, la représentation des travailleurs au sein de conseils d'administration ou l'autogestion ouvrière s.

Il va de soi que je ne puis entrer dans les détails et m'attarder sur les divers aspects de cette question, si complexe et si délicate, de la participation des travailleurs aux décisions. Toute généralisation sur un pareil sujet est nécessairement risquée, d'autant plus qu'il s'agit là d'un processus extrêmement dynamique susceptible d'un développement continu ainsi que de changements et d'adaptations constants. Les récentes législations adoptées en la matière par plusieurs pays européens en constituent des exemples frappants. Mais ce que je voudrais souligner, c'est le fait que le concept de participation - quelle que soit la difficulté d'en cerner le contenu avec précision - est probablement destiné à dominer l'évolution des relations professionnelles d'ici la fin de ce siècle.

$\mathrm{Si}$ je pense qu'il en sera ainsi, c'est essentiellement pour deux raisons : l'une, qui tient aux institutions et l'autre, aux hommes. Sur le plan des institutions, l'histoire des dernières années a mis en évidence l'importance de l'entreprise comme nœud de relations collectives entre employeurs et travailleurs. Sur le plan des hommes, il est non moins évident qu'en raison du développement et de la diffusion des connais- 
sances, le travailleur de 1974 , et spécialement le jeune travailleur, a des aspirations nouvelles que la présente organisation industrielle ne satisfait pas. Dans la Déclaration de Philadelphie, qui est annexée à la Constitution de l'OIT, il est écrit que «le travail n'est pas une marchandise » et c'est en partant de ce principe que toute l'action de l'OIT vise à améliorer le sort du travailleur et notamment ses conditions de travail, de manière que celles-ci respectent sa dignité et ne soient pas seulement dictées par des considérations d'ordre économique. Mais j'ai toujours pensé que ce principe avait une signification beaucoup plus large que la seule amélioration des conditions de travail. Il signifie aussi que le travailleur n'est pas un instrument, qu'il ne veut pas se sentir manipulé comme un outil, ni être la proie de forces ou l'enjeu de décisions sur lesquelles il n'a aucune prise.

Dans son rapport à la Deuxième Conférence régionale européenne, Wilfred Jenks écrivait: "Le travailleur européen d'aujourd'hui aspire à une plus grande liberté que celle que lui offre la société industrielle moderne - une plus grande liberté pour arranger sa vie à sa guise et une plus grande liberté à l'égard du nombre de contraintes que la société lui impose actuellement.

Le travailleur européen d'aujourd'hui aspire à un plus grand respect de sa dignité d'être humain que celui qu'il peut trouver dans un travail ennuyeux et monotone ou ressentir devant le rythme fiévreux et les impératifs de la vie de tous les jours.

Le travailleur européen d'aujourd'hui aspire à une plus grande part de responsabilité dans l'organisation de son existence quotidienne et de la vie de sa communauté que celle que lui permettent d'assumer les structures hiérarchiques rigides de l'entreprise ou les institutions dépersonnalisées de l'État-providence $>{ }^{2}$. Et il me semble trouver une préoccupation similaire dans le message du Ministre du Travail du Canada à l'occasion de la Fête du Travail lorsqu'il déclare: "There are, in Canada, far too many workers who suffer from feelings of impersonality and alienation induced by a workplace devised more for output than for human happiness $\$$.

Voilà pourquoi je pense que l'évolution vers une participation accrue des travailleurs et de leurs représentants au niveau de l'entreprise

2 Valeurs humaines et politique sociale. Rapport du Directeur général du BIT à la Deuxième Conférence régionale européenne, Genève, janvier 1974. BIT, Genève. p. 42. 
aux décisions qui affectent leur vie de travail se poursuivra et gagnera en importance. Mais la reconnaissance du principe que les travailleurs doivent participer collectivement à la solution des problèmes qui les concernent directement peut conduire en pratique à des solutions très différentes. Un premier type de solution, qui comporte d'ailleurs de nombreuses variantes, part du principe que l'influence des travailleurs doit s'exercer au sein des conseils d'administration ou de surveillance des entreprises, c'est-à-dire à la source même des décisions susceptibles d'affecter leurs intérêts, et que le droit de participation à la gestion, voire d'autogestion des entreprises, est un nouveau droit qui devrait désormais faire partie du statut du travailleur dans l'entreprise pour attester sa citoyenneté économique. Un deuxième type de solution refuse toute confusion entre le rôle de la direction - à qui il doit incomber de diriger l'entreprise avec les droits et les responsabilités que cette direction comporte - et le rôle des représentants des travailleurs, qui est de faire pression sur la direction pour que ceux-ci obtiennent le maximum d'avantages. À la conception de la participation au pouvoir de direction de l'entreprise, les partisans de ce deuxième type de solution - actuellement de beaucoup la plus répandue - opposent celle du contre-pouvoir, c'est-à-dire du pouvoir syndical extérieur à celui de la direction et destiné à lui faire contrepoids. La rencontre entre ces deux pouvoirs s'opère alors par l'intermédiaire de la négociation collective et du contrat collectif, dont le champ va d'ailleurs s'élargissant puisque, dans plusieurs pays, diverses questions qui, dans le passé, relevaient de décisions prises unilatéralement par les directions d'entreprises et appartenaient à la sphère réservée des «prérogatives de la direction > font actuellement l'objet de discussions paritaires. L'exemple de l'organisation du travail est, à cet égard, caractéristique.

Quel que soit le type de solution retenu, une des difficultés que soulève l'évolution des sociétés modernes est le conflit provoqué entre deux tendances qui vont dans des directions opposées. D'une part, la complexité et l'interdépendance des problèmes appellent des solutions à des niveaux toujours plus élevés, non seulement au plan d'un pays mais également au plan international (par exemple, en raison de l'existence du Marché commun) ou au plan multinational (du fait de l'existence de sociétés multinationales de plus en plus puissantes). D'autre part, les travailleurs entendent être associés à la prise des décisions qui doivent influer sur leurs emplois et leur vie de travail. L'aspiration vers une plus grande participation «à la base » rend plus difficile la prise des décisions «au sommet ». Elle n'en doit pas moins être considérée comme 
une nécessité inéluctable et la méconnaissance de cette aspiration à la participation en vue de gagner du temps ou d'éviter des difficultés immédiates risque fort d'être une politique à courte vue, susceptible d'engendrer à posteriori des conflits plus sérieux.

\section{Nécessité de justifier ses actes}

La nécessité de justifier ses actes n'est pas une notion nouvelle en matière de relations industrielles. Dans des pays comme le Canada ou les États-Unis, on a fait remarquer par exemple, que «l'introduction de la procédure de réclamation et le droit de recourir à l'arbitrage soumettent la direction à une sorte d'obligation de justifier son action quotidienne et transforment le caractère d'une direction privée en la faisant passer du stade du gouvernement autoritaire à celui d'un gouvernement constitutionnel ». L'introduction du principe de la justification $\mathrm{du}$ licenciement, auquel j'ai fait allusion plus haut, fournit un autre exemple du crédit croissant dont jouit cette idée que les actions privées susceptibles d'être suivies de répercussions sur le plan social peuvent avoir à être justifiées pour être acceptables. Pourquoi cette idée ne s'appliquerait-elle pas davantage aux actions collectives dans le domaine des relations professionnelles ? Pour répondre à cette question, il ne faut pas négliger le fait que, pendant les deux dernières décennies, un changement considérable s'est produit dans la conception du rôle dévolu au gouvernement dans le jeu des relations professionnelles. Il y a vingt ans, nombre de pays industrialisés acceptaient encore comme un article de foi le fait que l'harmonie des relations professionnelles dépendait avant tout du degré de développement des négociations collectives volontaires et que le rôle du gouvernement, essentiellement négatif, consistait à éviter de s'immiscer dans des affaires qu'il valait mieux laisser aux deux partenaires le soin de régler entre eux. Pour illustrer cette théorie, je rappellerai que, il y a dix-huit ans de cela, le très Honorable Sir Walter Monckton, alors ministre du Travail et du Service national de Grande Bretagne, prenant la parole au cours de la discussion du rapport du Directeur général à la session annuelle de la Conférence internationale $\mathrm{du}$ travail, soulignait que «la caractéristique fondamentale de notre politique tend à écarter de ces relations professionnelles et du travail quotidien, dans la mesure du possible, toute ingérence de l'État».

Il est vrai qu'en cas de rupture des négociations collectives, il était admis que le gouvernement se devait d'intervenir pour redresser la situation. Cependant, l'intervention gouvernementale avait un caractère essentiellement accessoire, son but étant d'aider les parties, par le 
déclenchement opportun de mécanismes de conciliation, d'enquête ou d'arbitrage volontaire, à remettre le train sur ses rails et non pas de le conduire ou d'en régler la vitesse. Même dans les pays où, comne aux Etats-Unis, on avait reconnu la nécessité d'une puissante intervention du législateur afin de garantir le droit de négociation collective, le but de cette législation, du moins à l'origine, était surtout de veiller à ce qu'une relation de négociation collective puisse s'établir. Tant que la paix sociale n'était pas sérieusement menacée, le gouvernement n'était pas intéressé au premier chef à l'issu des négociations collectives.

À l'heure actuelle, les parties à la négociation collective ne peuvent plus continuer à penser qu'elles sont absolument seules en cause. Pour reprendre les déclarations faites par un ancien Directeur général du BIT, M. David Morse, il y a quelques années : "Les gouvernements, dans leur politique de croissance économique, ne peuvent plus se satisfaire d'être les observateurs impartiaux du processus de la négociation collective. Ils doivent nécessairement prendre un intérêt direct à certaines des questions qui ont jusqu'ici été exclusivement du ressort de la négociation collective entre employeurs et travailleurs. Ce faisant, le gouvernement introduit dans le processus de négociation le concept de l'intérêt national ou public, qu'il considère comme sa responsabilité de définir et de faire valoir en plus des intérêts de groupes représentés par les organisations d'employeurs et de travailleurs ».

Cependant, il n'est pas facile de découvrir les solutions appropriées. On ne saurait oublier que le droit de s'organiser pour la protection des intérêts qu'il a en commun avec d'autres est une liberté fondamentale de l'homme, que les négociations collectives sont actuellement le meilleur moyen imaginé par le monde professionnel non seulement pour fixer les salaires et réglementer les conditions de travail, mais encore pour établir des relations harmonieuses entre les parties intéressées, et que de plus, au sein d'une société démocratique, les principes mis en œuvre pour améliorer le taux de croissnce et l'efficacité de l'économie ne peuvent être efficace qu'avec l'appui des employeurs et des syndicats ou, du moins, qu'à la condition de ne pas se heurter à leur opposition déclarée.

Le grand problème qui se pose actuellement en matière de relations professionnelles consiste par conséquent à déterminer comment les systèmes et les pratiques devraient être adaptés pour contribuer à atteindre des objectifs d'intérêt général sans pour cela mettre en péril l'autonomie et la liberté d'action des employeurs et des travailleurs. 
Lorsque je parle de la nécessité pour les parties aux négociations collectives de justifier leur comportement en tenant compte de l'intérêt général, je tiens à préciser que cette justification n'a pas à convaincre seulement le gouvernement - car une organisation syndicale peut par exemple avoir de bonnes raisons d'être opposée à la politique d'un gouvernement dont elle conteste les options économiques ou sociales mais aussi l'opinion publique. Avec le dévelopement des techniques d'information modernes, notamment audio-visuelles, les acteurs des relations industrielles, en cas de conflits d'importance, sont soumis au jeu des questions et le fait est que la réaction de l'opinion publique est un des facteurs essentiels dont ils doivent tenir compte pour déterminer leur conduite.

\section{La formation et la nécessité de l'éducation}

Le développement scientifique et technique, l'évolution des rapports économiques et sociaux, la nouveauté, la complexité et l'interdépendance des problèmes qu'ils soulèvent appellent un développement considérable des activités d'éducation et de formation, non seulement sur le plan strictement technique et professionnel, mais aussi sur le plan des connaissances économiques et sociales.

La Conférence internationale du travail sera saisie à sa prochaine session de projets de convention et de recommandation relatifs au congééducation payé - c'est-à-dire un congé accordé à un travailleur à des fins éducatives pour une période déterminée, pendant le temps normal de travail, sans perte de gains ou d'autres prestations - et ces projets soulignent que le principe du congé-éducation payé devrait être considéré comme un nouveau droit dans le domaine du travail répondant aux besoins réels de l'individu dans la société contemporaine.

La nécessité se fait également sentir d'accentuer le contenu social du programme de perfectionnement des cadres dirigeants des entreprises, et l'examen en profondeur des activités du BIT en ce domaine, entrepris par le Conseil d'administration du BIT à sa dernière session, a mis nettement ce point en lumière, lorsqu'il a notamment souligné les aspects sociaux de la responsabilité des cadres dirigeants en rappelant que nombreux sont les pays où l'on accorde à la contribution du monde des affaires à la vie de la société une importance croissante qui s'étend bien au-delà de la fourniture habituelle de biens et de services et où l'opinion publique, sinon la législation, tient les cadres dirigeants pour responsables d'un certain nombre de mesures qui englobent notamment le respect de l'environnement, les pratiques commerciales équitables, la loyauté en 
publicité, l'utilisation mesurée de l'énergie, la création d'emplois à l'intention de personnes et de groupes sociaux défavorisés, la contribution à l'éducation et au développement communautaire.

À ce propos - et ce sera ma conclusion - je voudrais vous citer des extraits de deux articles.

Dans l'un, paru dans «Fortune » en mars 1971, M. Jodson Gooding fait ressortir que dans les années 1950 et 1960 , le cadre admettait son rôle de serviteur des grandes sociétés, et bien que résolu à préserver son individualité, il cherchait à atteindre de concert avec la direction, les buts que l'entreprise jugeait valables. En revanche, les nouveaux venus requièrent de l'entreprise une réévaluation de sa politique et de ses objectifs. Il ajoutait qu'à l'École des Hautes Étude commerciales de Stanford, un groupe appelé Comité pour la responsabilité des sociétés avait engagé vingt pour cent des étudiants inscrits aux cours dans une campagne visant à encourager les chefs d'entreprise à prendre des mesures positives pour s'attaquer aux problèmes sociaux. Il concluait en soulignant que les sociétés ne peuvent plus compter sur une fidélité inconditionnelle de leurs cadres.

L'autre est un extrait du livre «Les jours qui viennent» dans lequel un industriel français, Pierre de Calan, exprime ses réflexions sur l'avenir de la société occidentale. "La crise actuelle de notre civilisation, écrit-il, exprime fondamentalement un déséquilibre: nous disposons de moyens surabondants au service de finalités incertaines ou médiocres. Pour endiguer et discipliner la puissance matérielle de l'homme, Bergson avait prédit que le monde aurait besoin d'un supplément d'âme. La puissance n'a pas cessé de croître : l'âme n'a pas suivi. Nous pouvons trop et nous ne voulons pas assez. Non que nous soyons devenus abouliques, mais nous ne possédons plus assez d'imagination pour concevoir des projets, plus assez de références pour les juger et les sélectionner, plus assez de foi pour y croire. ${ }^{3}$

\section{Industrial Relations : An International Viewpoint}

In view of the wide differences between industrial relations of the various countries of the world as well as between the political, economical and sociocultural factors which influence them, it might be thought that it is impossible to discern general trends from a comparative examination of industrial relations' phenomena and institutions.

3 Le Figaro, 24 janvier 1974, p. 10. 
This negative reaction, while perfectly correct in many respects, would not however correspond to the whole truth. Almost no country today lives in isolation. Ideas have no frontiers, in so far, of course, that there is freedom of expression. The tripartite meetings convened by the ILO offer a unique opportunity of discovering common problems and trends and their conclusions, especially when they take the form of Conventions and Recommendations, represent an international consensus on important problems of social policy.

Trends analysed in this article refer only to labour - management relations at the level of the undertaking and are mainly based on the experience of industrialised countries.

THE EVOLUTION OF THE INSTITUTIONAL FRAMEWORK OF LABOUR-MANAGEMENT RELATIONS AT THE LEVEL OF THE UNDERTAKING

Since the Second World War, three main trends have characterised this evolution. A first trend, under employers' influence, has placed the emphasis on the improvement of the social climate by stressing the common objectives which should be shared by all the members of an undertaking, and by seeking ways and means of enlisting the maximum contribution from each towards the common end. This search has been facilitated by advances in social sciences (studies on the motivations of the man at work) and in techniques of management (delegation of authority, management by objectives, etc..)

A second trend, essentially of a political nature, encompasses the developments which have changed the basic structure of the undertaking (nationalisations and various forms of workers' participation in the managerial bodies of public or private undertakings). The third trend consists of all the measures which, mainly under trade union influence, are concerned with regulating the behaviour of managements (by law, collective agreements or as result of day-to-day trade union action) in such a way as to curtail their freedom of action by means of a whole series of procedures and institutions. These regulations have resulted in nothing less than a Bill of Rights for workers, which has gradually been evolved to safeguard them in their dealings with their employers.

\section{THE CONTEMPORARY TRENDS}

Towards greater security. In a world which is rapidly changing it is not surprising that the aspiration for security should have become prominent in the industrial relations field. Thus an aspiration towards greater employment and income security is to be noted in most countries. The principle that a worker should not be arbitrarily deprived of his employment has been firmly recognised at the international level, when, in 1963, the International Labour Conference adopted the Recommendation (No. 119) on Termination of Employment, which states that «termination of employment should not take place unless there is a valid reason for such termination $»$. This is a very important change in comparison with the old philosophy according to which, when a worker has been recruited by an employer under a contract of indeterminate duration, this contract may be rescinded at the will of either party provided a certain period of notice is given. 
While the recognition of the principle of the justification of termination of employment is essential to ensure the dignity of the worker in the employment relationship, there is nothing demagogic in it. The "valid reason » which may justify termination of employment, may be «based on the operational requirements of the undertaking, establishment or service ». Management therefore remains free to manage according to the best interest of the undertaking. As it is increasingly recognised that technical progress and economic growth need a greater mobility of workers than in the past, security is not therefore synonymous of stability. Certain jobs become reduntant or obsolete. A particuliar job cannot therefore be protected at all costs. In such situations the trend towards greater security will focus on the protection of the workers' income and on the measures to be taken to facilities his reemployment. While reduction of workforce may be justified as a factor of economic progress, the workers affected by such reductions cannot be left on their own. If society is to benefit from economic progress, it has also to support the social cost of such progress. This is the new principle of social justice which is now gaining acceptance.

Towards greater participation. In 1966 the International Labour Conference adopted a Resolution noting that in various countries with different economic and social structures, efforts and experiments are being made to enable workers to participate in the decisions taken in their undertakings and requesting studies by the ILO on this subject. These studies, as well as various meetings organised by the ILO, have shown that while there cannot be an internationally agreed definition of the concept of «participation», workers are anxious to obtain a greater influence on the marking of the decisions which concern them, through methods as different as collective bargaining, joint consultation and communications, or workers' representation in the managerial boards. The concept of participation is likely to dominate the evolution of industrial relations in the next thirty years because the worker of today has new aspirations which are not satisfied by the present industrial organisation. $\mathrm{He}$ refuses to be manipulated like a tool or buffeted by forces or decisions over which he has no influence.

Towards greater accountability. Twenty years ago it was still an accepted credo in many industrialised countries that the role of government in industrial relations was mainly a passive one: not to interfere in matters which were best left to the autonomous relations of the two industrial partners. When collective bargaining broke, government intervention had mainly an auxiliary character. It was to assist the parties to put the train back on its rails, and not to drive it or to regulate its speed. So long as industrial peace was not seriously threatened, the government was not primarily concerned with the outcome of collective bargaining. Today the parties to collective bargaining can no longer consider that this is their own affair only. Governments in pursuing their economic and social policies are anxious to see the concept of the public interest introduced into the bargaining process. The finding of appropriate solutions is however not easy. The great challenges of our days in the industrial relations field is to determine how the systems and practices should be adapted in order to permit the achievement of public goals without endangering basic human freedoms. The partners to collective bargaining will have more and more to be in a position to justify their behaviour in the light of the public interest. 
The need for training and education. The complexity and interdependance of problems raised by technological developments and the evolution of economic and social relations call for a tremendous increase in training and education, not only from a technical point of view but also with regard to economic and social knowledge. The International Labour Conference will consider next June the drafts of a convention and a recommendation on educational leave for workers and the Governing Body of the ILO has emphasised the social responsibility of managers as one of the key elements of the future activities of the ILO Management Development Programme. This is all the more necessary that young executives are sometimes challenging the objectives pursued by the companies in which they work.

\section{LA POLITISATION DES RELATIONS DU TRAVAIL (28ème congrès 1973)}

Introduction, Gilles LAFLAMme - Les formes historiques de politisation du syndicalisme au Québec, Léo Roback - L'évolution socio-économique et le déplacement des centres de pouvoir, BERNARD SolaSSE - L'impact des secteurs public et para-public sur la politisation des relations du travail, JEAN Borvin - La philosophie du Code du travail, Jean-Réal Cardin - Les limites du négociable et le débordement des conflits, ANDRÉ THIBaUdeau - Positions des partis politiques devant la politisation des relations du travail, ROBERT Burns, ANdré Déom, Michel Bellavance - Conséquences de la politisation des relations du travail, GÉrard Dion - Annexe : Négociation collective dans un monde en évolution.

1 volume, 170 pages - Prix : $\$ 5.50$

\section{LES PRESSES DE L'UNIVERSITÉ LAVAL CITÉ UNIVERSITAIRE \\ Québec, P.Q., CANADA \\ G1K 7R4}

